\title{
Reduction of the blind spot in the time-frequency domain reflectometry
}

\author{
Ki Seok Kwak ${ }^{1}$, Seung Ho Doo ${ }^{1}$, Chun Ku Lee ${ }^{1}$, Jin Bae Park ${ }^{1 a)}$, \\ and Tae Sung Yoon ${ }^{2}$ \\ ${ }^{1}$ Department of Electrical and Electronic Engineering, Yonsei University, \\ Seoul, Korea \\ 2 Department of Electrical Engineering, Changwon National University, \\ 9 Sarim-dong, Changwon, Gyeongsangnam-do, Korea \\ a) jbpark@yonsei.ac.kr
}

\begin{abstract}
In this paper, we propose a method to reduce the blind spot based on signal processing that indicates the minimum length of a cable under test in the time-frequency domain reflectometry without using an extension cable or adding a new high-speed hardware component. The time-frequency domain reflectometry adopted the proposed method can be achieved with not only a simple modification of the previous system but also a simple technique based on signal processing. The experimental results show that the proposed method allows us to estimate fault distance on the cable with high spatial resolution.
\end{abstract}

Keywords: time-frequency domain reflectometry, blind spot, spatial resolution, fault detection

Classification: Science and engineering for electronics

\section{References}

[1] C. Furse and R. Haupt, "Down to the wire: The hidden hazard of aging aircraft wiring," IEEE Spectr., pp. 35-39, 2001.

[2] C. P. Nemarich, "Time domain reflectometry liquid level sensors," IEEE Instrum. Meas. Mag., vol. 4, pp. 40-44, 2001.

[3] G. Ripamonti, M. Ghioni, and A. Lacaita, "No dead-space optical timedomain reflectometer," J. Lightw. Technol., vol. 8, pp. 1278-1283, 1990.

[4] Product catalog for a time domain reflectometry [Online] http://www.tscm.com/riprcop.html

[5] J. F. Walsh, "Dual time base zero dead zone time domain reflectometer," U.S Patent 5440528, Aug. 8, 1995.

[6] R. Feced, M. Farhadiroushan, and V. A. Handerek, "Zero dead-zone OTDR with high-spatial resolution for short haul applications," IEEE Photon. Technol. Lett., vol. 9, pp. 1140-1142, 1990.

[7] Y. J. Shin, E. J. Powers, T. S. Choe, C. Y. Hong, E. S. Song, J. G. Yook, and J. B. Park, "Application of time-frequency domain reflectometry for detection and localization of a fault on a coaxial cable," IEEE Trans. Instrum. Meas., vol. 54, pp. 2493-2500, 2005.

[8] L. Cohen, "Time-frequency distributions-A review," Proc. IEEE, vol. 77, pp. 941-981, 1989. 


\section{Introduction}

The remarkable issues of aging electrical wiring, wire health monitoring system and associated faults in an aircraft have been emphasized in [1]. An electric arc that may result in serious damages to an overall system can be caused by a trivial fault on a wire. Therefore, detection, localization of fault with high accuracy and wire health monitoring system are highly demanded for diagnosis and maintenance of electric wiring systems.

A reflectometry instrument such as time domain reflectometry (TDR), time-frequency domain reflectometry (TFDR) and optical time domain reflectometry (OTDR) were originally developed to detect and localize faults along the cable or optical fiber. The TDR consists of sending a signal into the cable under test and measuring the reflected signal. An electrical signal launched onto the cable will be reflected at an impedance discontinuity point caused by changes in the electrical characteristic of the cable. The OTDR similarly measures and localizes the faults in an optical fiber. Therefore, the fault along the cable or optical fiber can be detected $[2,3]$.

However, it is well known that all the reflectometry methods have an inherited drawback known as a dead zone or a blind spot $[3,4,5,6]$. This blind spot takes place when the cable under test is short enough that the incident signal from a signal generator and the reflected signal from a fault overlap. It is a key specification of most reflectometry methods because it indicates the minimum length of the cable that can be measured.

There are some solutions to reduce the blind spot in the reflectometry methods. In the TDR, the dual time base technique and the addition of an extension cable were introduced in $[4,5]$. In the OTDR, the time-correlated single photon counting technique was presented in [6]. However, these solutions demand additional hardware configurations and have limitation that the extension cable should have the same impedance the characteristic as the cable under test. Any impedance variation due to the connection of the extension cable might cause an unexpected reflection.

The time-frequency domain reflectometry (TFDR) has been introduced and verified that it has an excellent performance than other conventional reflectometry methods to detect and localize faults on the cable [7]. However, it also has the blind spot since the incident signal called the reference signal overlaps with its reflected signal.

The blind spot in the TFDR is determined by the reference signal width. The wider the reference signal width is, the more reference signal energy is transmitted and will travel down a cable. Therefore, a tiny fault located at a long distance can be detected. To reduce the blind spot in the TFDR, the reference signal width should be narrow enough to avoid the overlap between the reference and the reflected signals. However, a very high speed signal generator is demanded for the TFDR to use the reference signal having narrow enough width. Moreover, the reference signal width gets narrower the reference signal energy may not be enough to travel down a cable and travel back. 
In this paper, we propose a method to reduce the blind spot without adding an extension cable or adapting a high speed hardware component in the TFDR. The experimental results show that the TFDR adopted the proposed method can detect and localize fault with high spatial resolution.

\section{The TFDR system and the blind spot}

The TFDR works on the similar principle as other conventional reflectometry methods except using the reference signal that is fitted in the time-frequency domain for the cable under test [7]. The reference signal from an arbitrary waveform generator (AWG) is generated and transmitted down the cable. When the reference signal reaches at the end of the cable or a fault on the cable, all or part of the reference signal is reflected back to the point where a digital storage oscilloscope (DSO) is. Figure 1 shows the proposed TFDR system configuration and its signal flow. The dotted lines imply very short connection and the bold line means the cable under test. The isolator helps the reflected signal from the fault reach to the DSO.

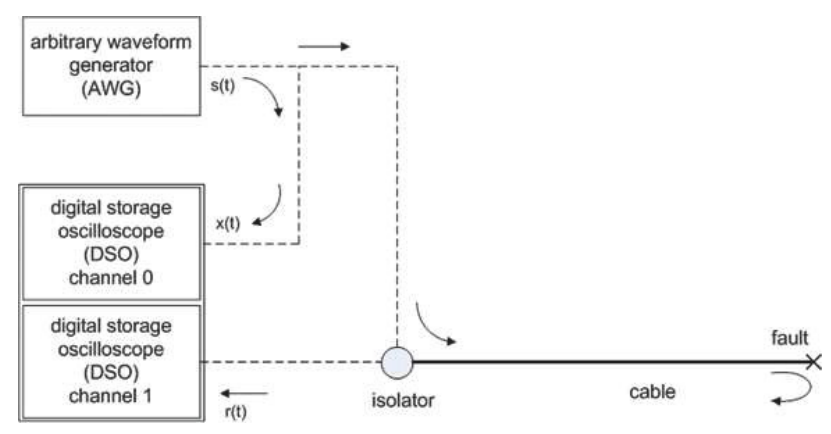

Fig. 1. The proposed TFDR system.

The blind spot $L_{B}$ in the TFDR can be determined by Eq. (1).

$$
L_{B}=\frac{v_{p} \times t_{\omega}}{2}
$$

where $v_{p}$ means the velocity of propagation in the cable, and $t_{\omega}$ is the reference signal width.

The TFDR uses a reference signal that is linearly modulated chirp signal with a Gaussian envelope. The reference signal can be written in Eq. (2).

$$
s(t)=\left(\frac{\alpha}{\pi}\right)^{1 / 4} e^{-\alpha\left(t-t_{0}\right)^{2} / 2+j \beta\left(t-t_{0}\right)^{2} / 2+j \omega_{0}\left(t-t_{0}\right)}
$$

where $\alpha, \beta, t_{0}$, and $\omega_{0}$ are the time duration, the frequency sweep rate, the time center, and the frequency center, respectively.

In respect of the signal width, the reference signal width gets wider, the more energy can be transmitted, then, much signal energy will travel down the cable. Therefore, a tiny fault located at a long distance can be detected and localized. On the other hand, the wider the reference signal width is, the longer the blind spot is. 
To reduce the blind spot, it is obvious that we should have $v_{p}$ or $t_{\omega}$ small in Eq. (1). Once the cable under test is chosen, the factor $v_{p}$ is determined by physical characteristic of the cable. The parameter $t_{\omega}$ can be simply reduced by a higher speed AWG. However, it might cost a lot.

In this paper, we suggest a method based on signal processing to reduce the blind spot in the TFDR without having a hardware component improved.

\section{The proposed method for reducing the blind spot}

When the cable under test is longer than the blind spot, the reference and the reflected signals are clearly separated. Then, we can calculate easily the fault distance with round-trip propagation time between two peaks in the timefrequency cross-correlation function such as shown in Fig. 2-(a) and (b). The third and the fourth peaks from the left side in Fig. 2-(a) imply that the reflected signal goes to the fault and returns back again. Therefore, we do not have to consider about the last two peaks because they are not directly related with the fault.

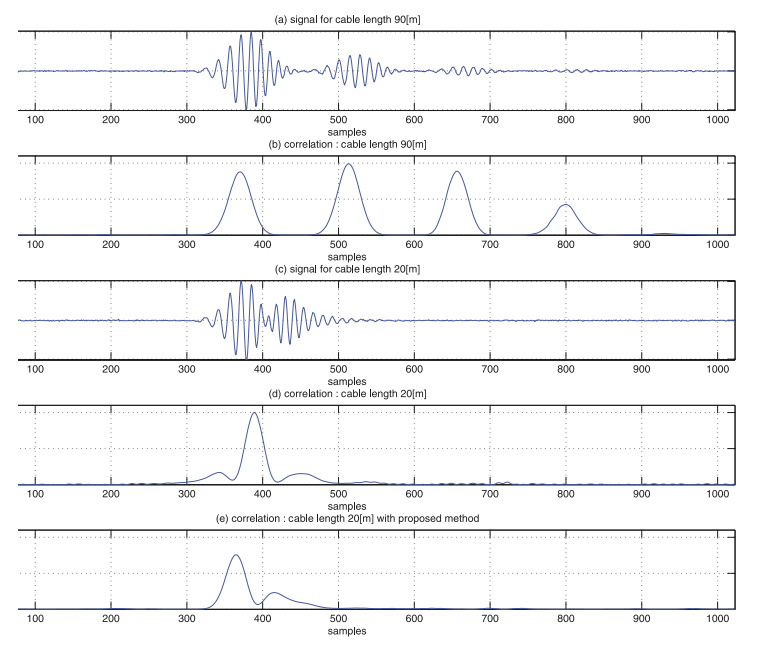

Fig. 2. The measurement signals and their timefrequency cross-correlations.

In the TFDR, the blind spot is seen when the width of the reference signal is wider than the time which the reflection occurs from a fault after injecting the reference signal along a cable. Then, the reference signal and the reflected signals overlap. Figure 2-(c) and (d) represent that the reference and the reflected signals overlap when a fault is located within the blind spot and their time-frequency cross correlation result, respectively. It seems that the reflected signal has just disappeared. Therefore, it is impossible to calculate the fault distance with round-trip propagation in the time-frequency crosscorrelation such as shown in Fig. 2-(b). When we can take the reflected signal out of the region where the reference and the reflected signals overlapped as shown in Fig. 2-(c), we may have the reference and the reflected signals separated, then we can calculate the fault distance via their time-frequency cross-correlation result. 
As shown in Fig. 1, an observation signal $x(t)$ from the AWG can be described as follows:

$$
x(t)=s(t)+\omega(t)
$$

where $s(t)$ is the designed reference signal and $\omega(t)$ means zero-mean Gaussian observation noise.

The reflected signal $r(t)$ from an impedance discontinuity point, i.e., a fault or end of target cable after injecting the designed reference signal can be defined by Eq. (4).

$$
r(t)=A s(t)+B s(t-\tau)+v(t)
$$

where $\tau$ is the time delay of the reference signal $s(t), A, B$ imply scaling factors, and $v(t)$ is zero-mean Gaussian observation noise.

Let us assume that the amplitude of the designed reference signal $s(t)$ is normalized. The scaling parameter $A$ has the range of $0<A<1$ by considering of connection cable's attenuation characteristic. The scaling parameter $B$ has the range of $0<B<1$ by considering of the target cable's attenuation characteristic since the reflected signal energy might be relatively small comparing to the normalized reference signal. We can define that the time delay parameter $\tau \neq 0$ since we consider the reflection should take place somewhere in the cable under test. Then, we can simply obtain the reflected signal component using Eq. (5) after multiplying $1 / A$ by Eq. (4) and subtracting it from Eq. (3).

$$
\frac{B}{A} s(t-\tau)=\frac{1}{A} r(t)-s(t)-\omega^{\prime}(t)
$$

where $\omega^{\prime}(t)$ is $\frac{1}{A} v(t)$.

\section{Results and discussion}

We set an experimental TFDR system as shown in Fig. 1 using an AWG with $200 \mathrm{M}$ samples/sec, a DSO with $200 \mathrm{M}$ samples/sec, and an isolator. The reference signal used in this experiment had the width of 700 [ns] and the frequency bandwidth of $10 \sim 20[\mathrm{MHz}]$. We selected a target cable 10CFBT having a fault, open status, that the velocity of propagation is known to be 83.4 percent of the light speed. Therefore, the blind spot will be $89.25[\mathrm{~m}]$.

The aim of this experiment is to show that the TFDR adopting the proposed method can detect and localize when the cable under test is shorter than the blind spot. We acquired the reference and the reflected signal such as shown in Fig. 2-(a) and (c) via the TFDR, then the time-frequency cross correlation between the reference signal and the reflected signal was calculated after eliminating the overlap by using the proposed method to estimate the fault distance. In Fig. 2-(b), the time-frequency cross-correlation result was obtained when the target cable was longer than the blind spot. The fault distance was estimated as $90.0714[\mathrm{~m}]$ since the time indices of the two peaks were 370 and 514 , respectively.

The signal in Fig. 2-(c) was obtained when the cable under test was $20[\mathrm{~m}]$, which was shorter than the blind spot. Therefore, the reference and the 
reflected signals overlapped. The fault distance was not able to be estimated without using the proposed method since there was one peak at time index 390 as shown in Fig. 2-(d). Note that we obtained the result as shown in Fig. 2-(e) by adopting the proposed method. Since the time indices of the two peaks were 370 and 404, the estimated fault distance was $21.2668[\mathrm{~m}]$.

The experimental results are represented in Table I. The results show that the proposed method can reduce the blind spot in the TFDR. However, the error rate increases when the region that the reference and the reflected signals overlap gets increased.

Table I. Experimental results.

\begin{tabular}{|c|c|c|}
\hline cable length $[\mathrm{m}]$ & estimated distance $[\mathrm{m}]$ & error rate [\%] \\
\hline 90 & 90.0714 & 0.0793 \\
\hline 80 & 80.0634 & 0.0793 \\
\hline 70 & 70.0555 & 0.0793 \\
\hline 60 & 60.0476 & 0.0793 \\
\hline 50 & 50.0396 & 0.0792 \\
\hline 40 & 40.6572 & 1.6430 \\
\hline 30 & 31.9003 & 6.3343 \\
\hline 20 & 21.2668 & 6.3340 \\
\hline 10 & 8.1314 & 18.6860 \\
\hline 5 & 6.2550 & 25.1000 \\
\hline
\end{tabular}

\section{Conclusion}

In this paper, we achieved to reduce the blind spot in the TFDR using the proposed method based on simple signal processing instead of using a higher speed hardware component. Therefore, the proposed method can be a cost effective solution. Moreover, it does not cause any unexpected reflection due to adding an extension cable. The experimental results show that it is possible for the TFDR adapted the proposed method to detect and localize the fault distance with high spatial resolution.

\section{Acknowledgments}

This research was funded by the Korea Electrical Safety Corporation (KESCO), Project \#R-2006-1-229, "Implementation of real-time fault monitoring system for transmission line." Also this work was supported in part by Yonsei University Institute of TMS Information Technology, a Brain Korea 21 program and in part by MOCIE through EIRC program with Yonsei Electric Power Research Center (YEPRC) at Yonsei University, Seoul, Korea. 